\title{
Ride Sharing Attitudes Before and During the COVID-19 Pandemic in the United States
}

\author{
Parastoo Jabbari' ${ }^{1}$, Don MacKenzie ${ }^{2}$ () \\ ${ }^{1}$ Department of Civil and Environmental Engineering, University of Washington (WA), ${ }^{2}$ Department of Civil and Environmental Engineering, University of \\ Washington \\ Keywords: covid-19, travel behavior, transit ridership, ride-sharing \\ https://doi.org/10.32866/001c.17991
}

Findings

\begin{abstract}
We explore how the COVID-19 pandemic has changed individuals' attitudes and perceptions toward sharing rides. In 2019, the year before the pandemic, we had implemented an online survey to understand US travelers' attitudes and perceptions of modes, including transit and pooled ride-hailing. During the pandemic, in May 2020, we redistributed a portion of that survey to the same respondents. We compare the distributions of responses from before and during the pandemic and test the significance of these shifts. We found that while people's dislike of sharing rides with strangers has not changed compared with before the pandemic, their willingness to share to save money has significantly changed. Also, more people are not OK with crowded buses and try to use modes that allow them to avoid others.
\end{abstract}

\section{Research Question and Hypothesis}

The COVID-19 pandemic and resulting stay-at-home orders and closures of nonessential businesses drastically changed travel choices and options available. Ride-hailing services eliminated pooling options, transit ridership fell significantly, and traffic jams disappeared - temporarily. While actions by governments and employers have driven many of these shifts, individuals' attitudes toward some transportation modes, specifically those requiring sharing of confined spaces, may have changed as well. In this paper we test whether the pandemic changed individuals' attitudes toward sharing rides with others.

\section{Methods and Data}

In May 2020, we redistributed part of a national survey that we had previously deployed in 2019, to the same pool of participants. The repeated questions related to people's attitudes and perceptions toward modes that require sharing of vehicles with strangers. We tested whether there was a significant change in reported behaviors and attitudes after the onset of the pandemic.

In 2019, we used Amazon Mechanical Turk (MTurk) to recruit participants. Each MTurk survey taker has a unique Worker ID which was used to limit survey participation to individuals who had taken our survey in 2019. From 591 participants who were eligible based on participation in the 2019 survey, 277 individuals responded to the 2020 survey. The survey included sociodemographic questions and psychometric measures related to sharing 
Table 1: Responses to "What is your current employment status?"

\begin{tabular}{|l|r|}
\hline 2020 Employment status $(\mathbf{n}=277)$ & $76.7 \%$ \\
\hline Full-time & $10.7 \%$ \\
\hline Self-employed & $6.4 \%$ \\
\hline Part-time & $3.9 \%$ \\
\hline Unemployed & $1.1 \%$ \\
\hline Retired & $0.3 \%$ \\
\hline Homemaker & \\
\hline
\end{tabular}

rides. We kept the wording of the 2020 survey questions the same as the 2019 questions. All respondents reported being at least 18 years old when taking the first survey.

The mean and median ages were 38 and 36 years old, respectively, during the 2020 survey. U.S. national median age was 38.2 in 2018 (US Census Bureau 2019). Our sample is slightly younger than general population. Sample median household income falls into the $\$ 40,000-\$ 60,000$ income bracket. U.S. national median income in 2019 was $\$ 68,703$ (US Census Bureau 2020). Our sample includes individuals from larger metropolitan areas and smaller cities.

$55 \%$ of the survey respondents were male and $45 \%$ female. Table 1 summarizes self-reported employment status in the 2020 survey. $77 \%$ of participants indicated that they have a full-time job.

\section{Findings}

In the 2020 survey, we included four statements related to sharing rides and asked respondents to indicate what they feel about these statements. Then we compared responses collected during the pandemic with responses collected a year before, in May 2019. Figure 1 shows the results. We used a Wilcoxon signed-rank test to test whether the shifts we observed in the 2020 responses are statistically significant. The Wilcoxon signed-rank test tests whether the differences in the mean ranks between pairs of observations are statistically significant (Kraska-Miller 2013).

The first statement targets how participants feel about sharing a ride with strangers. The general feeling is "uncomfortable" both before and during the pandemic. We observed minor shifts from "slightly uncomfortable" to "uncomfortable". However, the changes in responses are not statistically significant based on the Wilcoxon signed-rank test $(p=0.4)$. Respondents were already uncomfortable sharing rides with strangers, and that has not changed because of the pandemic.

The second statement was "I would like to share rides with others if it saves money" and participants were asked to indicate their level of agreement or disagreement. As shown in Figure 1, there was a shift from agreeing with the 

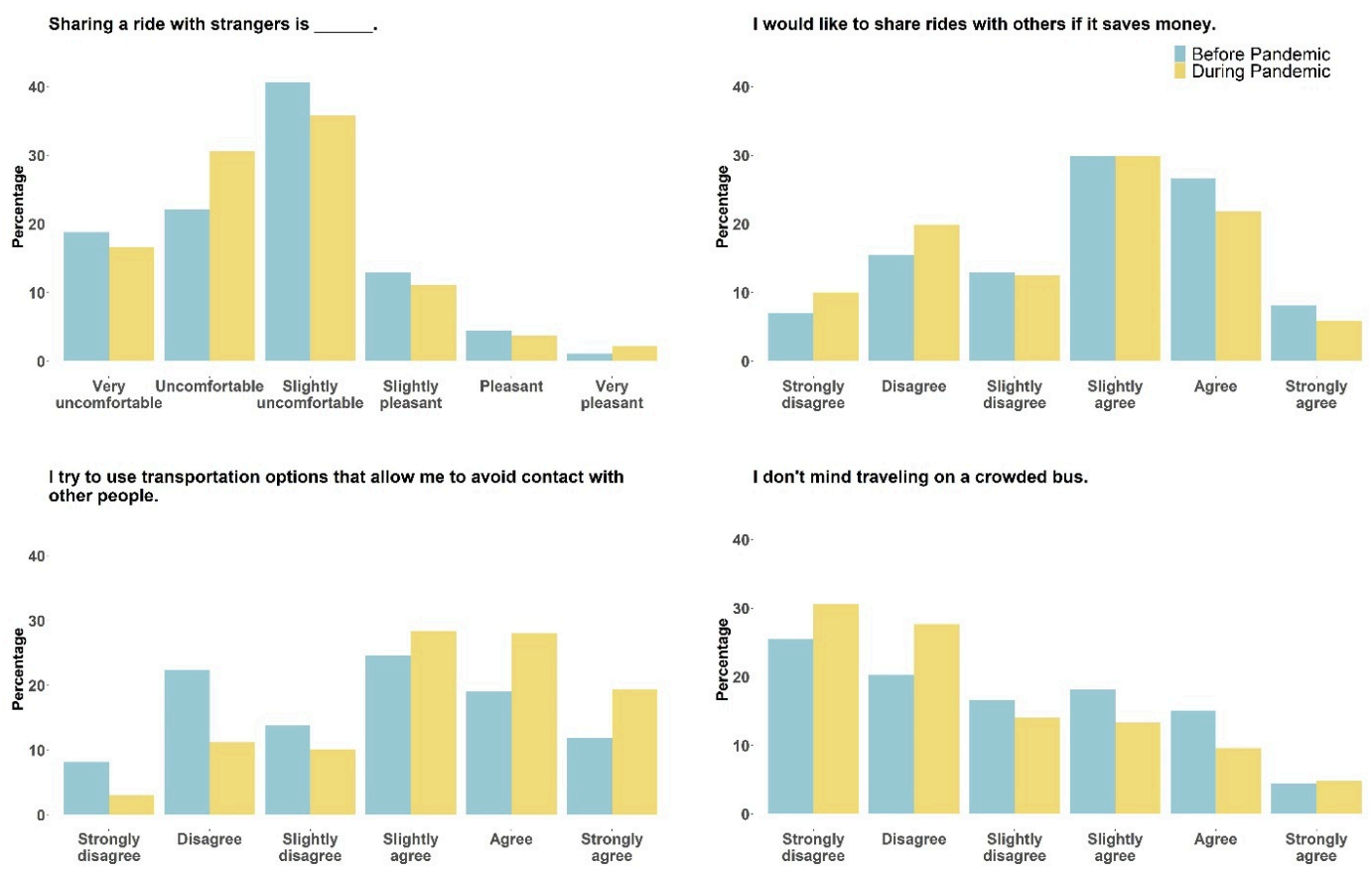

Figure 1: Responses to survey statements before and during the pandemic. Upper left: Sharing a ride with strangers is [very uncomfortable... very pleasant]. Upper right: I would like to share rides with others if it saves money [strongly disagree... strongly agree]. Lower left: I try to use transportation options that allow me to avoid contact with other people [strongly disagree... strongly agree]. Lower right: I don't mind travelling on a crowded bus [strongly disagree... strongly agree].

statement toward disagreeing, during the pandemic. The Wilcoxon signedrank test indicates that the difference in the two samples is statistically significant $\left(p=3.4 \times 10^{-3}\right)$. This suggests that the tradeoff between sharing rides and money has changed during the pandemic and individuals are less willing to share rides to save money compared with before the pandemic.

We observed a sizable shift in reported responses for the statement "I try to use transportation options that allow me to avoid contact with other people." During the pandemic people reported stronger levels of agreement with this statement and based on the Wilcoxon signed-rank test these changes are statistically significant $\left(p=1.02 \times 10^{-9}\right)$. This is consistent with what is expected during the pandemic as Americans were advised to keep at least 6 feet $(1.8 \mathrm{~m})$ apart to reduce disease transmission.

The last statement specifically targeted individuals' attitudes toward crowded buses. Even though we observed that responses in both time periods are more concentrated in disagreement categories, during the pandemic the disagreement is stronger. The Wilcoxon signed-rank test $\left(p=2.3 \times 10^{-4}\right)$ confirms that during the pandemic significantly more individuals had a problem with crowded buses compared with before the pandemic. 


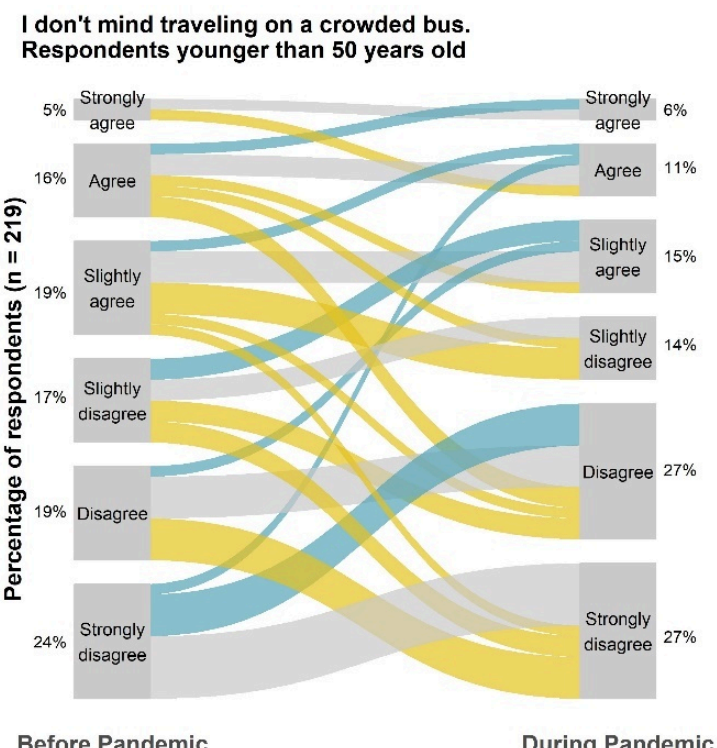

Before Pandemic

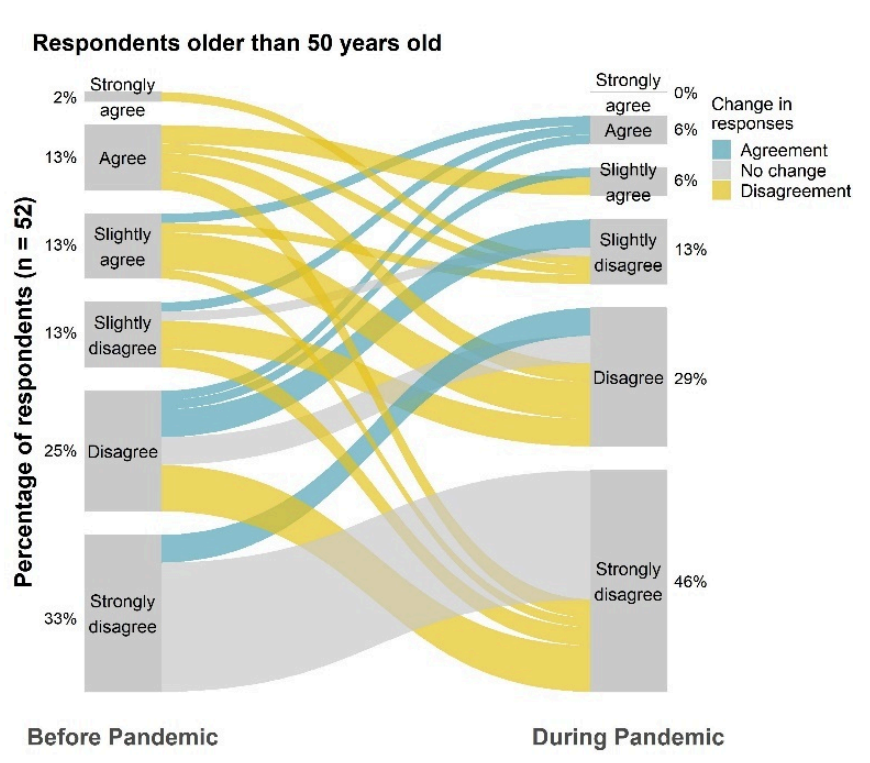

Figure 2: Mapping of individual responses before the pandemic to same individual's response during the pandemic for the statement "I don't mind traveling on a crowded bus".

Based on our data, we can see that people were already uncomfortable sharing rides with others before the pandemic, and that has not changed much. What has changed is that respondents are now less willing to put that discomfort aside to save money. This has implications for ridesharing companies and transit agencies as they are already struggling to keep pooled ride services economically sustainable.

The COVID-19 disease poses a greater danger to older individuals and those with pre-existing medical conditions. We broke down our sample by age, to explore whether we observe different attitudes among older individuals. Figure $\underline{2}$ shows that during the pandemic $46 \%$ of individuals over 50 years of age indicated that they strongly disagreed with the statement that "I don't mind traveling on a crowded bus". The figure also shows that older individuals shifted more strongly toward disagreement than did younger respondents. The fact that we do not observe more drastic changes in responses is due at least in part to the fact that older individuals disliked crowded buses even before the pandemic. 


\section{REFERENCES}

Kraska-Miller, M. 2013. Nonparametric Statistics for Social and Behavioral Sciences. CRC Press.

US Census Bureau. 2019. "Income and Poverty in the United States: 2019.” The United States Census Bureau. https://www.census.gov/library/publications/2020/demo/p60-270.html.

- - . 2020. "Median Age Doesn't Tell the Whole Story.” The United States Census Bureau. https://www.census.gov/library/stories/2019/06/median-age-does-not-tell-the-whole-story.html. 


\section{SUPPLEMENTARY MATERIALS}

\section{Data}

Download: https://findingspress.org/article/17991-ride-sharing-attitudes-before-and-during-thecovid-19-pandemic-in-the-united-states/attachment/47423.csv

\section{Metadata}

Download: https://findingspress.org/article/17991-ride-sharing-attitudes-before-and-during-thecovid-19-pandemic-in-the-united-states/attachment/47424.txt 